\title{
Mesolimbic Functional Magnetic Resonance Imaging Activations during Reward Anticipation Correlate with Reward-Related Ventral Striatal Dopamine Release
}

\author{
Björn H. Schott, ${ }^{1,2,3 *}$ Luciano Minuzzi, ${ }^{4 *}$ Ruth M. Krebs, ${ }^{2}$ David Elmenhorst, ${ }^{4}$ Markus Lang, ${ }^{5}$ Oliver H. Winz, ${ }^{4}$ \\ Constanze I. Seidenbecher, ${ }^{1}$ Heinz H. Coenen, ${ }^{5}$ Hans-Jochen Heinze, ${ }^{1,2}$ Karl Zilles, ${ }^{4}$ Emrah Düzel, ${ }^{2,6^{\ddagger}}$ and \\ Andreas Bauer ${ }^{4} 7^{\ddagger}$ \\ ${ }^{1}$ Leibniz Institute for Neurobiology, 39118 Magdeburg, Germany, ${ }^{2}$ Department of Neurology, Otto von Guericke University of Magdeburg, 39120 Magdeburg, \\ Germany, ${ }^{3}$ Department of Psychiatry, Campus Mitte, Charité University Hospital, 10117 Berlin, Germany, ${ }^{4}$ INB-3 (Medicine) and ${ }^{5}$ INB-4 (Nuclear Chemistry), \\ Institute of Neurosciences and Biophysics, Research Center Jülich, 52425 Jülich, Germany, ${ }^{\top}$ Institute of Cognitive Neuroscience, University College London, London \\ WC1N 3AR, United Kingdom, and ${ }^{7}$ Department of Neurology, Heinrich Heine University Düsseldorf, 40225 Düsseldorf, Germany
}

The dopaminergic mechanisms that control reward-motivated behavior are the subject of intense study, but it is yet unclear how, in humans, neural activity in mesolimbic reward-circuitry and its functional neuroimaging correlates are related to dopamine release. To address this question, we obtained functional magnetic resonance imaging (fMRI) measures of reward-related neural activity and $\left[{ }^{11} \mathrm{C}\right]$ raclopride positron emission tomography measures of dopamine release in the same human participants, while they performed a delayed monetary incentive task. Across the cohort, a positive correlation emerged between neural activity of the substantia nigra/ventral tegmental area (SN/VTA), the main origin of dopaminergic neurotransmission, during reward anticipation and reward-related $\left[{ }^{11} \mathrm{C}\right] \mathrm{ra}$ clopride displacement as an index of dopamine release in the ventral striatum, major target of SN/VTA dopamine neurons. Neural activity in the ventral striatum/nucleus accumbens itself also correlated with ventral striatal dopamine release. Additionally, high-reward-related dopamine release was associated with increased activation of limbic structures, such as the amygdala and the hippocampus. The observed correlations of reward-related mesolimbic fMRI activation and dopamine release provide evidence that dopaminergic neurotransmission plays a quantitative role in human mesolimbic reward processing. Moreover, the combined neurochemical and hemodynamic imaging approach used here opens up new perspectives for the investigation of molecular mechanisms underlying human cognition.

Key words: dopamine; fMRI; reward; midbrain; substantia nigra; nucleus accumbens; neurotransmission; receptor

\section{Introduction}

Reward processing in the mammalian brain depends critically on the interaction of the ventral striatum/nucleus accumbens (NAcc), and the midbrain dopaminergic system, particularly the medial substantia nigra and ventral tegmental area (SN/VTA). A key mechanism in mesolimbic reward processing is the phasic activity of dopamine-releasing SN/VTA neurons in response to unexpected rewards and reward-predicting cues (reward antici-

\footnotetext{
Received May 5, 2008; revised 0ct. 1, 2008; accepted Nov. 10, 2008.

This work was supported by the German Helmholtz Association (Virtual Institute VH-VI-125), the Deutsche Forschungsgemeinschaft (SFB 779, TP A8 and KFG 163, TP3), the "Pakt für Forschung und Innovation," and the State of Saxony-Anhalt. We gratefully acknowledge the excellent technical assistance of W. Roden and S. Stüsgen (Institute of Nuclear Chemistry), M. Vogeling (Molecular Neuroimaging Group), K. Frey, L. Tellmann, E. Theelen, and H. Herzog (PET Instrumentation Group), B. Elghahwagi, P. Engels, G. Oefler, and C. Kemper (MRI Instrumentation Group, Research Center Jülich), and Denise Göttert and Claus Tempelmann (Center of Advanced Imaging, University of Magdeburg).

The authors declare no competing financial interests.

*B.H.S. and L.M. contributed equally to this work.

${ }_{E}^{\ddagger} E$.D. and A.B. share senior authorship.

Correspondence should be addressed to Dr. Björn H. Schott, Department of Behavioral Neurology, Leibniz Institute for Neurobiology, Brenneckestrasse 6, 39118 Magdeburg, Germany. E-mail: bschott@neuro2.med.uni-magdeburg.de. D01:10.1523/JNEUROSCI.2058-08.2008

Copyright $\odot 2008$ Society for Neuroscience $\quad$ 0270-6474/08/2814311-09\$15.00/0
}

pation) (Schultz, 1998). The NAcc is a major target of these midbrain dopaminergic projections. Accordingly, human functional neuroimaging studies have shown activations of the SN/VTA and the ventral striatum during reward anticipation (Knutson et al., 2001; Knutson and Cooper, 2005; Wittmann et al., 2005).

The functional magnetic resonance imaging (fMRI) signal does not provide direct information about neurochemistry, and it remains thus unclear how event-related mesolimbic fMRI activation relates to striatal dopamine release. This principal limitation of fMRI remains despite attempts to improve its spatial resolution for the VTA (D'Ardenne et al., 2008) and requires comparative use of more direct measures of activity-dependent dopamine release. Positron emission tomography (PET) studies using the $\mathrm{D}_{2 / 3}$ receptor ligand $\left[{ }^{11} \mathrm{C}\right]$ raclopride, which is displaceable by endogenous dopamine, have demonstrated striatal dopamine receptor binding potential $\left(\mathrm{BP}_{\mathrm{ND}}\right)$ reductions during rewarded tasks. In a pioneering study, Koepp et al. (1998) showed that activity-dependent striatal $\left[{ }^{11} \mathrm{C}\right]$ raclopride displacement during a rewarded video game could be measured in vivo. Zald et al. (2004) showed, more specifically, that rewarded tasks were associated with a stronger $\left[{ }^{11} \mathrm{C}\right]$ raclopride $\mathrm{BP}_{\mathrm{ND}}$ decrease than nonrewarded tasks with comparable sensorimotor activity. 
Pharmacological MRI studies suggest a positive relationship between striatal dopamine levels and hemodynamic reward responses (Pessiglione et al., 2006; Knutson and Gibbs, 2007). A recent study on placebo-driven dopamine release showed a positive correlation between individual striatal dopamine release and reward-related NAcc activation (Scott et al., 2007). However, tasks in the two imaging modalities were not matched, and the analysis was restricted to the ventral striatum. Therefore, the question of how activity in the SN/VTA, where the perikarya of the dopamine-releasing neurons are located, is related to rewardrelated striatal dopamine release remains open.

In the present study we sought to directly investigate the relationship between reward-related striatal dopamine release and the fMRI correlates of reward anticipation. We correlated reward-related $\left[{ }^{11} \mathrm{C}\right]$ raclopride $\mathrm{BP}_{\mathrm{ND}}$ reduction and the $\mathrm{fMRI}$ signal during reward anticipation across a cohort of 11 young, healthy human volunteers. Subjects performed a delayed monetary incentive task in which reward-predicting or neutral cues were followed by a reaction time task upon which reward was instrumental (Wittmann et al., 2005; Schott et al., 2007). Because of the slow kinetics of $\left[{ }^{11} \mathrm{C}\right]$ raclopride binding, the task was divided into a rewarded (75\% reward trials) and a nonrewarded ( $0 \%$ reward trials) session, which were performed on 2 consecutive days. To allow for better comparability of the fMRI and PET sessions, the fMRI study was also divided into two days, and the statistical model of the fMRI data analysis was adapted accordingly (see Materials and Methods). We hypothesized that fMRI activation of the SN/VTA and of the NAcc during reward anticipation would be positively correlated with reward-related striatal dopamine release.

\section{Materials and Methods}

\section{Participants}

Fourteen young (age range 20-25 years, mean $22.8 \pm 1.5$ ) healthy volunteers participated in the experiment, three of which had to be excluded from analysis due to excessive movement in the PET study (two cases) or not complying with the task instructions (one case). All underwent routine clinical interview for neurological or psychiatric disorders and had normal T1-weighted MR images. Exclusion criteria were present or past neurological or psychiatric diseases and the use of centrally acting drugs, including regular nicotine use (two subjects were light social smokers according to self report). Subjects were asked to avoid the intake of nicotine and alcohol for at least $24 \mathrm{~h}$ and caffeine for at least $12 \mathrm{~h}$ before the measurements. They were also instructed to wake up in the morning at the same time on the days of the experiments to control diurnal variations in dopamine functioning. All participants gave written informed consent to participate in the study, in accordance with the Declaration of Helsinki. The PET protocol had been approved by the Ethics Committee of the Medical Faculty of the Heinrich Heine University Düsseldorf as well as the German Federal Institute for Drugs and Medical Devices and the German Federal Office of Radiation Protection. The fMRI study was performed in accordance with the guidelines of the Ethics Committee of the University of Magdeburg, Faculty of Medicine.

\section{Experimental paradigm}

We chose a variant of the delayed monetary incentive task (Knutson et al., 2001; Wittmann et al., 2005) as experimental paradigm, in which participants were conditioned to specific stimulus-reward associations. Thus, we aimed to avoid potential training effects over sessions, which might have been observable in prediction error-based learning tasks. The paradigm consisted of two types of sessions (reward vs neutral), which were performed on separate days. The same paradigm was used for both PET and fMRI studies, with the session order and stimulus material counterbalanced across subjects. The only difference between PET and fMRI study designs was that in PET, stimulation was performed for 36 min without interruption, while in fMRI, the paradigm was, on both days, split into three sessions of 12 min each (Fig. 1, top).

The trial structure for rewarded and neutral trials is depicted in Figure 1 (bottom). At the beginning of each trial, subjects saw a colored photograph of an indoor or outdoor scene for $3500 \mathrm{~ms}$ that served as a cue picture indicating the possibility for a reward or the absence of a reward in the following number comparison task, respectively. For half of the subjects outdoor scenes served as reward-predicting cues, for the other half indoor scenes. In the rewarded condition, 135 rewarded and 45 nonrewarded trials were presented, and subjects were told that they could win money, or lose a smaller amount if they responded incorrectly or too slowly, in the rewarded trials, whereas the responses in the neutral trials would not influence their overall gain. To minimize differences in sensorimotor and cognitive processing between the rewarded and neutral conditions, the neutral condition contained 135 trials consisting of neutral cues followed by neutral outcome and 45 trials consisting of cues from the rewarded category, which were also followed by neutral feedback (i.e., bogus reward trials). Apart from this, the trial structure was kept the same as in the rewarded condition. Subjects were explicitly told this, and were instructed to respond via button press whether they saw an indoor or outdoor scene.

Each cue picture was followed by a simple number comparison task (Pappata et al., 2002; Wittmann et al., 2005), in which subjects had to respond to a briefly flashed number $(100 \mathrm{~ms})$ ranging from 1 to 9 (except 5). Subjects were asked to press the left button for numbers below 5 and the right button for numbers over 5 within a certain time window. In rewarded trials, the subject received a green arrow as positive feedback for correct and fast responses, indicating a gain of 50 cents. Whenever the response was incorrect or too slow, a red arrow was displayed on the screen, indicating a loss of 20 cents. In nonrewarded trials, a question mark served as a neutral feedback, regardless of the correctness of the response. To obtain comparable hit rates across subjects, the response time window for the incentive task was obtained during the training phase before scanning, and was subsequently adjusted automatically for every new trial based on the preceding individual performance and reaction time of each subject. This dynamical adjustment led to a mean hit rate of $\sim 80 \%$ for each subject. The feedback delay was jittered between 0 and $6 \mathrm{~s}$ to separate activations depending on reward anticipation and reward outcome, respectively. Thus, the total trial length varied between 8 and $12 \mathrm{~s}$. Additionally, cued fixation periods lasting up to $18 \mathrm{~s}$ were included at randomly selected time points to allow for proper baseline estimation in the fMRI study (see below). To familiarize subjects with the upcoming task and reduce learning effects during the actual scanning, subjects completed a 5 min training session before the actual experiment. Training was repeated on each scanning day (reward vs neutral $\times$ PET vs fMRI) to condition subjects to the upcoming task and to reduce negative prediction errors to unrewarded "reward" cues (see above). Prediction error-based learning was thus minimized during the actual scanning, and the dopaminergic response could be expected upon presentation of reward-predicting cues (Schultz, 1998).

\section{Positron emission tomography study}

$\left.{ }^{11} \mathrm{C}\right]$ Raclopride synthesis. Raclopride and norraclopride (free base) were purchased from ABX. Methanol was dried by distillation from magnesium turnings under argon. Li-methylate was obtained from Aldrich. All other chemicals and solvents were purchased from Merck and were used as delivered by the vendor. The benzamide $\left[{ }^{11} \mathrm{C}\right]$ raclopride was prepared at high specific radioactivity as described previously (Stüsgen et al., 2007). Briefly, no-carrier-added $\left[{ }^{11} \mathrm{C}\right]$ methyl iodide was synthesized according to a published procedure (Holschbach and Schüller, 1993). Analytical radio-HPLC used a Kromasil $100-5$ C18 column $(250 \times 4.6$ $\mathrm{mm})$. Isocratic elution with $\mathrm{CH}_{3} \mathrm{CN} / 0.006 \mathrm{~N} \mathrm{H}_{3} \mathrm{PO}_{4}+\mathrm{NaH}_{2} \mathrm{PO}_{4} \times$ $2 \mathrm{H}_{2} \mathrm{O}(7.9 \mathrm{~g} / \mathrm{L}$ eluent), 35/65 (v/v), was at a flow rate of $1 \mathrm{ml} / \mathrm{min}$. UV monitoring at $210 \mathrm{~nm}$ detected raclopride and side products. For measurement of radioactivity, the outflow of the UV detector was connected in series to an on-line $\mathrm{NaI}(\mathrm{Tl})$ well-type scintillation detector. Chromatograms were corrected for the transit time between the detectors. Semipreparative HPLC was performed on a Kromasil 100-5 C18 column $(250 \times 8 \mathrm{~mm})$. Isocratic elution with $\mathrm{CH}_{3} \mathrm{CN} / 0.006 \mathrm{~N} \mathrm{H}_{3} \mathrm{PO}_{4}+$ 


\section{PET}

fMRI

\section{Reward condition}

\section{1 session (36 min)}

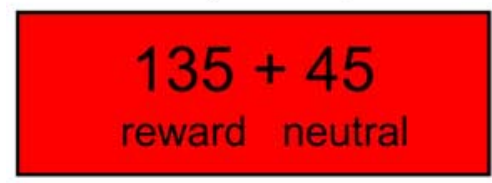

3 sessions (12 min each)

$45+15$

reward neutral

\section{Neutral condition}

1 session (36 min)

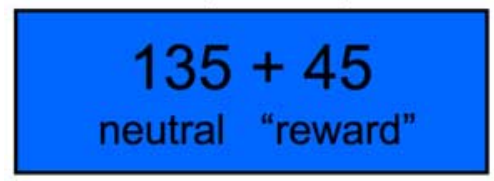

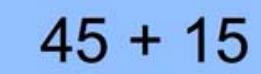

neutral "reward"
3 sessions (12 min each)

\section{Reward trial}

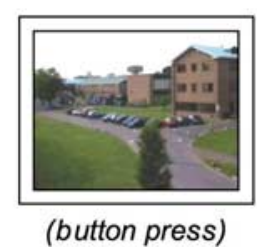

(button press)

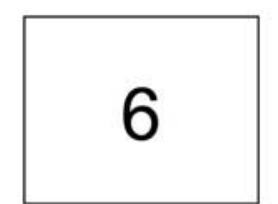

(button press)
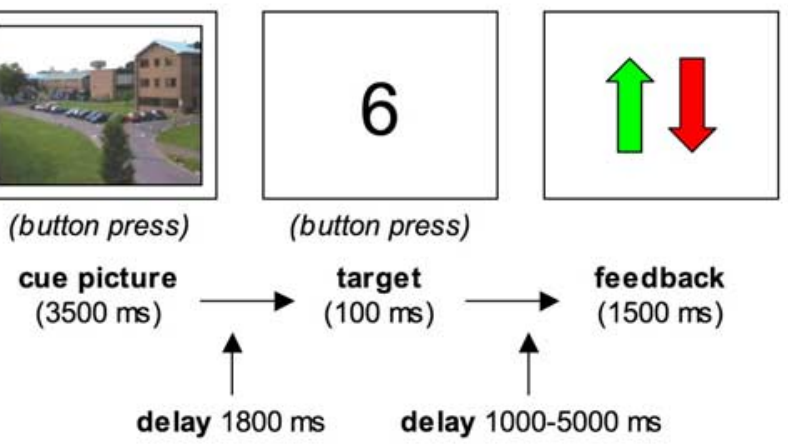

\section{Neutral trial}

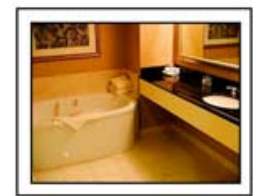

(button press)

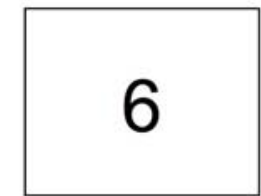

(button press)
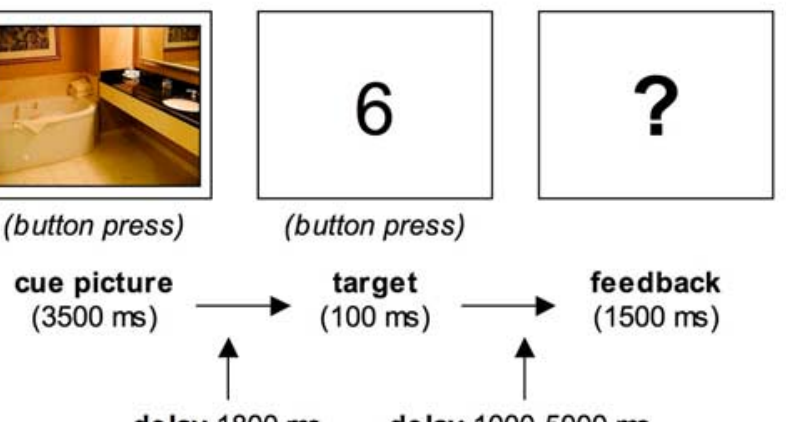

delay $1800 \mathrm{~ms}$

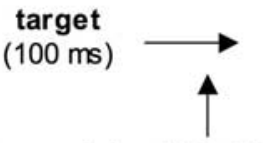

delay $1000-5000 \mathrm{~ms}$

Figure 1. Schematic illustration of the experimental paradigm. Top, Both the PET and the fMRI experiment consisted of a rewarded (135 rewarded trials, 45 neutral trials) and a neutral condition (135 neutral trials and 45 bogus reward trials), which were conducted on 2 consecutive days, counterbalanced across participants. The "reward" trials in the neutral condition consisted of reward-predicting cue pictures, followed by a neutral feedback. Bottom, The trials consisted of a cue picture indicating the possibility of a reward (indoor or outdoor scenes, counterbalanced across participants), followed by a target number and a feedback after a variable delay ranging from 1000 to $6000 \mathrm{~ms}$. The feedback was positive or negative in the rewarded trials (arrow up or down) and neutral ("?") in the neutral trials.

$\mathrm{NaH}_{2} \mathrm{PO}_{4} \times 2 \mathrm{H}_{2} \mathrm{O}(22.6 \mathrm{~g} / \mathrm{L}$ eluent $), 28 / 72(\mathrm{v} / \mathrm{v})$, was at a flow rate of 5 $\mathrm{ml} / \mathrm{min}$ with UV detection at $210 \mathrm{~nm}$. For solid phase extraction Sep Pak cartridges (Waters Oasis HLB $60 \mathrm{mg}$ ) were preconditioned with EtOH $(10 \mathrm{ml})$ and water $(10 \mathrm{ml})$. The lithium phenoxide precursor-salt was prepared by dissolving norraclopride (the free amine) in three molar equivalents of $0.1 \mathrm{M}$ methanolic LiOMe followed by evaporation to dryness under a stream of helium. The formed glassy solid of the lithium phenolate was dissolved in dimethylformamide $(500 \mu \mathrm{l})$ and immediately subjected to methylation $\left(80^{\circ} \mathrm{C}, 3 \mathrm{~min}\right)$ followed by helium gas purge $\left(80^{\circ} \mathrm{C}, 2 \mathrm{~min}\right)$. The reaction mixture was subjected to semipreparative HPLC, the fraction containing the product was collected, diluted with water $(90 \mathrm{ml})$, and purified by solid-phase extraction. Elution of the tracer with $\mathrm{EtOH}$, dilution with isotonic saline and filtration through a sterile filter gave $\left[{ }^{11} \mathrm{C}\right] \mathrm{ra}-$ clopride ready for injection.

PET scanning protocol. To exclude abnormalities in the CNS and to perform the coregistration of the anatomical data with the PET results, a high-resolution MRI was acquired from each subject using a Siemens 1.5T Magnetom Vision scanner in a 3D T1-weighted magnetizationprepared rapid acquisition gradient-echo sequence. The subjects were conducted to the PET scanner facility around $1 \mathrm{~h}$ before the injection of the radioligand. A training session lasting $5 \mathrm{~min}$ was applied before every scan. The subjects were subsequently placed in supine position with their heads being fixated in canthomeatal orientation by a vacuum pad. Head position was continuously monitored by a video system and reference skin marks, and manually corrected, if necessary. A venous catheter was installed in the arm of the subjects for the radioligand administration.

Dynamic PET recordings were acquired in 3D mode with the Siemens ECAT Exact HR+ tomograph. Before injection of the radiotracer, a $10 \mathrm{~min}{ }^{68} \mathrm{Ge} /{ }^{68} \mathrm{Ga}$ transmission scan was acquired to correct for attenuation. A 60-minlong dynamic emission recording was initiated upon intravenous bolus injection of $\left[{ }^{11} \mathrm{C}\right] \mathrm{ra}-$ clopride (mean injected radioactivity = $243.7 \pm 33.87 \mathrm{MBq}$ in the rewarded condition and $234.3 \pm 22.87 \mathrm{MBq}$ in the neutral condition; $t=0.563, p=0.586$ ) over $1 \mathrm{~min}$. PET data were acquired in list mode and reframed into the dynamic sequence of $6 \times 5 \mathrm{~s}, 3 \times 10 \mathrm{~s}, 4 \times$ $60 \mathrm{~s}, 2 \times 150 \mathrm{~s}, 2 \times 300 \mathrm{~s}$, and $4 \times 600 \mathrm{~s}(\mathrm{Lam}-$ mertsma and Hume, 1996).

Image processing and generation of binding potential maps. Individual MRI datasets were realigned manually to the anterior commissure/ posterior commissure line using interactive three-dimensional image registration software (MPI-Tool version 3.35, ATV) (Pietrzyk et al., 1994). Summed PET recordings for each subject were manually coregistered to the individual realigned MRI datasets and the registration parameters were applied to each dynamic frame using MPI-Tool software. Individual anatomical volumes of interest defining caudate (total, lateral, and medial), putamen (total, lateral, and medial), nucleus accumbens, and cerebellum were drawn by freehand onto the individual MRI images using PMOD software (version 2.75).

$\mathrm{BP}_{\mathrm{ND}}$ is defined as the ratio of the specifically bound to the nondisplaceable radioligand in the tissue at equilibrium (Innis et al., 2007). Parametric maps of $\left[{ }^{11} \mathrm{C}\right]$ raclopride $\mathrm{BP}_{\mathrm{ND}}$ were calculated using the noninvasive method of Logan (Logan et al., 1996) implemented in PMOD software, with the cerebellum as reference region. 


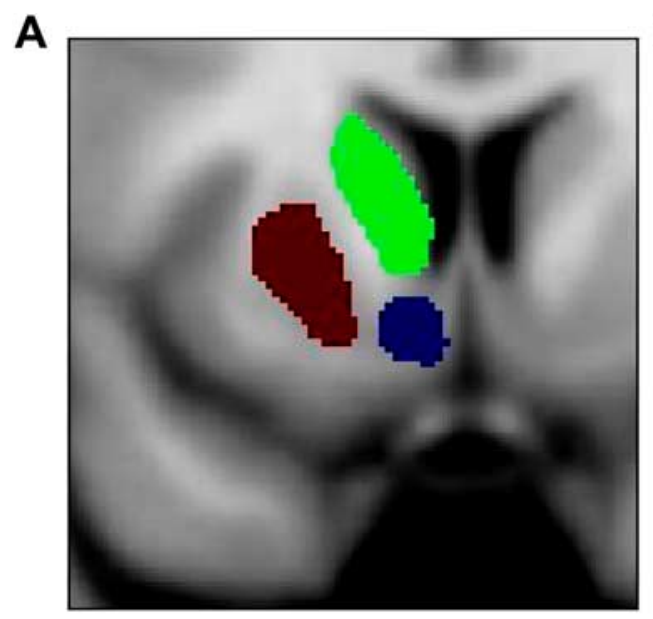

B

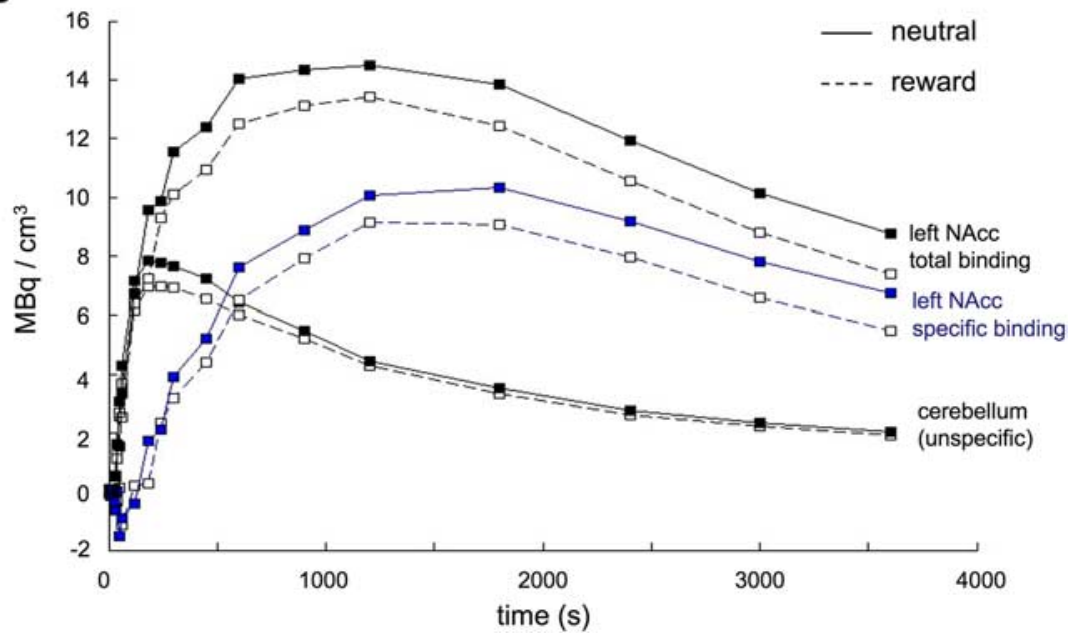

Figure 2. Reward-related $\left[{ }^{11} \mathrm{C}\right] \mathrm{raclopride}$ displacement. $\boldsymbol{A}$, Coronal section of the striatal ROls in the left hemisphere. The corresponding ROIs in the right hemisphere were also segmented. $\boldsymbol{B}$, Time-activity curves of a representative single subject. Total binding in the NAcc, unspecific binding in the cerebellum, and specific binding in the NAcc as the difference NAcc-cerebellum are shown. The $x$-axis depicts the time of PET scanning, starting with the injection of [11C]raclopride. The $y$-axis displays local radioactivity in the NAcc and cerebellum and the difference of NAcc and cerebellum activity, in $\mathrm{MBq} / \mathrm{mm}^{3}$.

Statistical analysis. Statistical analyses were performed using SPM2 (Statistical Parametric Mapping, Wellcome Department of Cognitive Neurology) and the MarsBaR region of interest (ROI) analysis tool (Brett et al., 2002).

For voxel-based analysis, individual $\mathrm{BP}_{\mathrm{ND}}$ parametric maps were initially smoothed with a Gaussian kernel of $3 \times 3 \times 3 \mathrm{~mm}$ and coregistered to the subjects' individual proton density (PD)-weighted MR images acquired during the fMRI study (see below). $\mathrm{BP}_{\mathrm{ND}}$ maps were then normalized into a standard stereotactic reference space [Montreal Neurological Institute (MNI)] and smoothed using a Gaussian kernel of $6 \times$ $6 \times 6 \mathrm{~mm}$. The striatum was segmented manually into caudate, putamen, and nucleus accumbens bilaterally from a normalized $(1 \times 1 \times 1$ $\mathrm{mm})$ and smoothed $(3 \times 3 \times 3 \mathrm{~mm}) \mathrm{PD}$-weighted MR image of one of the study participants (female, 24 years), using the MRIcro image analysis software tool (http://www.sph.sc.edu/comd/rorden/mricro.html). A representative coronal section depicting the resulting normalized anatomical ROIs is shown in Figure $2 \mathrm{~A}$. Statistical analysis over these ROIs was performed using the MarsBaR ROI analysis tool and a paired $t$ test model, comparing the reward condition and the neutral condition for each subject. The significance threshold for the ROI analysis was set to $p=0.05$, Bonferroni-corrected for the number of ROIs. For voxelwise analysis, the same statistical model was applied, and the significance threshold was set to $p=0.005$, uncorrected, with a minimum of 15 adjacent voxels, and the analysis was restricted to the striatum.

\section{Functional MRI study}

Image acquisition. Functional magnetic resonance imaging was performed using a Siemens Trio 3T magnetic resonance system and a standard head coil. Three sessions of 360 echoplanar images (EPIs) were acquired in an interleaved manner [32 axial slices; voxel size $=3.5 \times$ $3.5 \times 2 \mathrm{~mm} ; \mathrm{TR}=2 \mathrm{~s} ; \mathrm{TE}=30 \mathrm{~ms}$; even numbers first $]$. Additionally, a coplanar PD-weighted MR image (voxel size $=0.88 \times 0.88 \times 2 \mathrm{~mm}$; $\mathrm{TR}=6820 \mathrm{~ms}$; $\mathrm{TE}=14 \mathrm{~ms}$ ) was obtained and used for coregistration to improve spatial normalization.

Data processing and analysis. As in the PET study, data analysis was performed using SPM2. EPIs from both scanning days were corrected for acquisition delay, realigned to the first image acquired (starting with the images acquired on the first scanning day, regardless of session type, i. e. reward vs no reward), and the coplanar PD-weighted image was coregistered to the mean image obtained from realignment. For spatial normalization to the MNI reference frame (voxel size $=2 \times 2 \times 2 \mathrm{~mm}$ ), the coplanar PD-weighted image was used to determine normalization parameters, as $\mathrm{PD}$-weighted images exhibit a pronounced signal in the substantia nigra (D'Ardenne et al., 2008), thus improving normalization results in the midbrain. To verify normalization in the brainstem, normalized coplanar PD-weighted images were visually compared with a normalized mean PD-weighted image from five healthy male volunteers (age range, 18-27 years), who did not participate in the current study (data available upon request). Normalized EPIs were smoothed using a Gaussian kernel of $6 \times 6 \times 6 \mathrm{~mm}$.

Statistical analysis was performed using a two-stage mixed-effects model. In the first stage, the hemodynamic response was modeled by convolving a delta function at stimulus onset with a canonical hemodynamic response function (Friston et al., 1998). The resulting time courses were downsampled for each scan to form covariates of a general linear model (GLM). The model included separate covariates for each of the conditions of interest (rewarded condition: reward-predicting and neutral cues feedback to correct and false responses to reward-predicting and neutral cues, respectively, and target numbers; nonrewarded condition: neutral cues, bogus reward cues, neutral feedback to correct and false responses, respectively, and target numbers). The nonrewarded "reward" cues in the neutral condition were included as a separate covariate as they might be associated with larger negative prediction errors than the neutral cues. The six rigid-body movement parameters determined from realignment were included in the GLM as covariates of no interest. Model estimation was performed using an ordinary least squares fit, and contrasts of parameter estimates were computed for the hemodynamic responses to reward anticipation. To allow for a better comparability with the PET model (which compared sessions from two separate days), contrasts of the parameter estimates for reward anticipation included the reward cues (regardless of outcome, weighted +1 ) and the neutral cues (weighted -0.25) from the rewarded condition, as well as the neutral cues in the nonrewarded condition (weighted -0.75 ). This way, cues were weighted according to their proportions of occurrence in the rewarded and neutral condition [i. e. on the two scanning days]. Previous studies had shown the possibility of between-session comparisons in fMRI, when the inclusion of null events/fixation periods allows for a proper baseline estimation (Josephs and Henson, 1999; Schott et al., 2005). In the second stage of the model, these contrasts were submitted to a random-effects analysis, treating each subject as a random effect. Specifically, one-sample $t$ tests were computed over images of the reward anticipation contrasts. As in the PET study, the significance threshold was set to $p<0.005$, uncorrected, with a minimum of 15 adjacent voxels.

Correlational analysis of $F M R I$ and PET data. As the primary aim of our study was the identification of BOLD indices of endogenously released dopamine, fMRI correlates of reward anticipation were compared with $\left[{ }^{11} \mathrm{C}\right]$ raclopride $\mathrm{BP}_{\mathrm{ND}}$ reduction in the rewarded relative to the nonre- 
warded condition, rather than to total $\left[{ }^{11} \mathrm{C}\right]$ raclopride $\mathrm{BP}_{\mathrm{ND}}$ in either condition. Guided by our a priori hypothesis that reward-related dopamine release would be most prominent in the ventral striatum and by the PET results showing the most reliable reward-related $\left[{ }^{11} \mathrm{C}\right]$ raclopride displacement in the left NAcc (confirmed by both regional and voxelwise analysis), we chose the $\mathrm{BP}_{\mathrm{ND}}$ decrease in this region as independent variable for a linear regression analysis.

Based on the similarity between response patterns of midbrain dopamine neurons in animals (Schultz, 1998) and the human NAcc (Knutson and Cooper, 2005) and on the previously observed modulation of ventral striatal prediction errors by dopaminergic medication (Pessiglione et al., 2006), we hypothesized that reward-related $\left[{ }^{11} \mathrm{C}\right]$ raclopride displacement would be correlated with fMRI activations of the dopaminergic midbrain and potentially also of the ventral striatum. We therefore conducted ROI analyses in these brain regions. Guided by previous studies, in which midbrain activity that was likely to reflect the activity of dopaminergic neurons has typically been localized to the SN/VTA (Wittmann et al., 2005, 2007; Adcock et al., 2006; Bunzeck and Düzel, 2006; Schott et al., 2006, 2007), we segmented this region from a normalized PDweighted MR image, separately for the left and right hemispheres (Schott et al., 2006; Ahsan et al., 2007), using the MRIcro software. In the ventral striatum, $\mathrm{T}^{*}$ signal loss in basal forebrain regions, which was highly variable across subjects, and the resulting interindividual variability in ventral striatal fMRI activations had to be accounted for. Therefore, instead of using a common segmented ROI, individual spherical ROIs in the ventral striatum were selected for each subject by seeding a sphere (radius $=6 \mathrm{~mm}$ ) at the individual local maxima of the reward anticipation contrast closest to $[x y z]=\left[\begin{array}{lll}-6 & 10 & -6\end{array}\right]$, the coordinate where the PET analysis had shown the maximal radioligand displacement in the rewarded compared with the nonrewarded condition.

The ROI analysis was performed as a two-stage mixed-effects model, using SPM2 and the MarsBaR ROI analysis tool. In the first stage, individual GLMs were computed over the mean signal intensities of the ROIs for each subject, using the same conditions of interest and covariates as in the voxelwise contrasts (see above). In the second stage, a linear regression analysis was performed over the midbrain and NAcc contrast values of the reward anticipation contrast, using $\left[{ }^{11} \mathrm{C}\right]$ raclopride displacement in the left NAcc (neutral - rewarded) as independent variable. The significance threshold for the correlations was set to $p<0.05$, one-tailed, as positive correlations between dopamine release and mesolimbic BOLD signals were hypothesized.

To perform an exploratory analysis of additional fMRI activations that might correlate with reward-related dopamine release, a voxelwise linear regression was performed over the individual subjects' contrast maps derived from the voxelwise SPM analysis (see above), also using $\left[{ }^{11} \mathrm{C}\right] \mathrm{ra}-$ clopride displacement in the left NAcc as independent variable. As in all voxelwise comparisons, the significance threshold was set to $p<0.005$, uncorrected, with an extent threshold $k=15$ adjacent voxels.

\section{Results}

\section{Behavioral results}

Across both the PET study and the fMRI study, subjects reliably recognized reward cues and neutral cues (mean correct response rates $>0.92$ for all categories), although correct cue responses were slightly higher in the rewarded relative to the unrewarded session, with lowest hit rate occurring for the bogus reward cues in the nonrewarded condition (0.921 in fMRI and 0.933 in PET). An ANOVA for repeated measures over modality (PET vs fMRI), condition (rewarded versus unrewarded), and trial type (reward cues vs neutral cues) revealed a main effect of condition $\left(F_{(1,10)}=\right.$ $25.36, p=0.001)$ and a condition $\times$ trial type interaction $\left(F_{(1,10)}\right.$ $=16.38, p=0.002)$, but no effect of modality and no further interactions (all $p>0.123$ ). Subjects responded correctly to the target numbers in all conditions (all mean correct response rates $>0.96)$.

Table 1 displays the reaction times (RTs) to cue pictures and target numbers, separated by modality, condition, and trial type.
Table 1. Reaction times to reward cues and target numbers, separated by modality

\begin{tabular}{llllll}
\hline Condition & Reward & & & Neutral & \\
Trial type & Reward & Neutral & & "Reward" & Neutral \\
\hline Cues & & & & \\
$\quad$ PET & $694 \pm 142$ & $766 \pm 113$ & & $808 \pm 170$ & $775 \pm 165$ \\
$\quad$ fMRI & $614 \pm 120$ & $708 \pm 110$ & & $784 \pm 181$ & $715 \pm 137$ \\
Targets & & & & \\
$\quad$ PET & $454 \pm 40$ & $501 \pm 47$ & & $516 \pm 61$ & $513 \pm 59$ \\
$\quad$ fMRI & $412 \pm 30$ & $454 \pm 46$ & & $487 \pm 61$ & $483 \pm 59$ \\
\hline
\end{tabular}

Mean reaction times (in $\mathrm{ms}, \pm S \mathrm{~S}$ ) are shown for the responses to cue pictures (i. e. reward-predicting and neutra scenes) and to target numbers, separately for the PET and fMRI study.

RTs were fastest for the reward trials in the rewarded condition [main effect of condition: $F_{(1,10)}=8.89, p=0.014$; interaction condition $\times$ trial type: $F_{(1,10)}=81.80, p<0.001$; three-way ANOVA for repeated measures (modality $\times$ condition $\times$ trial type)]. Similarly, the responses to targets were fastest to rewarded targets in the rewarded condition [main effect of session type: $F_{(1,10)}=22.01, p=0.001$; main effect of trial type: $F_{(1,10)}=81.47$, $p<0.001$; condition $\times$ trial type interaction: $F_{(1,10)}=39.90, p<$ 0.001 ; three-way ANOVA for repeated measures (modality $\times$ condition $\times$ trial type)]. Because rare items (neutral cues in the rewarded session and "reward" cues in the unrewarded session) were associated with longer reaction times, we computed post hoc paired $t$ tests over the RTs to reward cues and targets from the reward session and the neutral cues and targets from the nonrewarded session, separately for PET and fMRI. In all cases, shorter RTs for the reward condition were observed (all $t_{(10)}>2.05$, all $p<0.034)$. Across sessions and conditions, RTs were slightly longer in the PET experiment (cues: main effect of modality: $F_{(1,10)}=28.96, p<0.001$; targets: main effect of modality: $F_{(1,10)}$ $=54.94, p<0.001$ ), possibly due to the more distracting environment in the PET relative to the fMRI experiment.

\section{Reward-related $\left[{ }^{11} \mathrm{C}\right.$ ]raclopride displacement}

Subjects showed a significant decrease of $\left[{ }^{11} \mathrm{C}\right]$ raclopride $\mathrm{BP}_{\mathrm{ND}}$ in the left ventral striatum during the rewarded condition (i.e., $75 \%$ of reward trials) compared with the nonrewarded condition (i.e., no reward trials) (Fig. 1), most likely resulting from ligand displacement by endogenous dopamine (Koepp et al., 1998). Figure $2 \mathrm{~B}$ depicts representative $\left[{ }^{11} \mathrm{C}\right]$ raclopride $\mathrm{BP}_{\mathrm{ND}}$ curves for specific radioligand binding in the ventral striatum as difference between total NAcc binding and nonspecific binding in the cerebellum from a single study participant. A ROI-based analysis of striatal $\left[{ }^{11} \mathrm{C}\right]$ raclopride $\mathrm{BP}_{\mathrm{ND}}$ reduction using anatomical ROIs segmented from a normalized $\mathrm{PD}$-weighted MR image (Fig. 2) revealed a robust $\mathrm{BP}_{\mathrm{ND}}$ decrease in the left NAcc that remained significant after Bonferroni correction for the number of ROIs (Table 2). Voxelwise $t$ test statistics confirmed this result by revealing a significant cluster of $\mathrm{BP}_{\mathrm{ND}}$ reduction in the left NAcc $[p<0.005$, uncorrected; extent threshold $k=15$ adjacent voxels (Fig. 3, left)].

\section{Functional MRI correlates of reward anticipation}

To allow for better comparability with the PET results, the fMRI experiment was also performed on two consecutive days (rewarded vs unrewarded sessions). Rewarded trials were compared with control trials of both the rewarded and the neutral condition (weighted 1:3, proportionally to the total number of cues; see Materials and Methods for details). In line with previous studies, reward anticipation was associated with activation of an extensive mesolimbic network. Compared with neutral cues, rewardpredicting cues were associated with an increased $\mathrm{fMRI}$ response 
Table 2. $\left[{ }^{11} \mathrm{C}\right]$ raclopride displacement in the striatum

\begin{tabular}{|c|c|c|c|c|}
\hline & $\mathrm{BP}_{\mathrm{ND}}$ decrease & SPM contrast value & $T$ & $p$ \\
\hline \multicolumn{5}{|c|}{ Nucl. accumbens } \\
\hline Left & $0.17 \pm 0.075$ & 0.08 & 2.86 & $0.0084^{*}$ \\
\hline Right & $0.02 \pm 0.075$ & 0.00 & 0.00 & 0.4986 \\
\hline \multicolumn{5}{|l|}{ Caudate } \\
\hline Left & $0.04 \pm 0.045$ & 0.03 & 1.03 & 0.1646 \\
\hline Right & $-0.03 \pm 0.194$ & -0.03 & -1.24 & 0.8792 \\
\hline \multicolumn{5}{|l|}{ Putamen } \\
\hline Left & $0.01 \pm 0.088$ & 0.01 & 0.23 & 0.4098 \\
\hline Right & $-0.05 \pm 0.062$ & -0.00 & -0.08 & 0.5309 \\
\hline
\end{tabular}

The table displays $B P_{N D}$ decreases in the rewarded relative to the nonrewarded condition. $B P_{N D}$ reductions in the individual anatomical voxels of interest from the PMOD analysis (means \pm SEs) and statistical results from the MarsBaR-based analysis of the normalized ROIs in SPM2 are shown. *The result in the left NAcc remained significant at $p<0.05$ after Bonferroni correction for the number of ROls.

of the ventral (and, to some extent, dorsal) striatum (Fig. 3, right), the insula, and of the anterior midbrain (SN/VTA). Moreover, several limbic structures, including the amygdala, the hippocampus, and the rostral anterior cingulate exhibited higher BOLD responses for reward-predicting compared with neutral cues.

There was no activation in these regions in response to predicted reward outcome (i.e., positive vs neutral feedback). This is compatible with the possibility that positive prediction errors in our paradigm were small in magnitude given that the reward cues predicted the value of the rewards well (the expected value of the reward cues was 0.35 cents, and the reward magnitude was 0.50 cents) (Knutson et al., 2001; Knutson and Cooper, 2005; Wittmann et al., 2005; Schott et al., 2007).

\section{Correlation of PET and $\mathrm{fMRI}$ data}

While previous studies have established relationships between striatal D2 receptor $\mathrm{BP}_{\mathrm{ND}}$ and cognitive performance (Cervenka et al., 2008) or fMRI activation patterns (Heinz et al., 2004), the variable of interest in the present study was the endogenously released dopamine. Therefore, $\left[{ }^{11} \mathrm{C}\right]$ raclopride $\mathrm{BP}_{\mathrm{ND}}$ difference between the neutral and rewarded conditions, which is likely to result from increased dopamine release during reward processing (Koepp et al., 1998), was chosen as independent variable for the correlational analyses. To investigate how the hemodynamic reward anticipation response corresponds to reward-related dopamine release, a linear regression analysis of the dopamine $\mathrm{BP}_{\mathrm{ND}}$ reduction in the left NAcc and the fMRI signal was computed. Because of our a priori hypothesis that SN/VTA activation would reflect the activity of dopaminergic neurons, we conducted a ROI analysis of the midbrain. For the ROI analysis in the midbrain, the left and right SN/VTA were segmented manually from a normalized and smoothed PD-weighted MR image of a study participant. According to visual inspection, this ROI corresponded well to the individual SN/VTA in the normalized PD-weighted images of all study participants. Across the 11 subjects $\left[{ }^{11} \mathrm{C}\right]$ raclopride displacement in the left NAcc showed a significant positive correlation with the hemodynamic response of the left SN/VTA $(r=$ $0.748, p=0.004$ ) (Fig. 4 , top). There was also a positive, but statistically nonsignificant, correlation of left NAcc $\left[{ }^{11} \mathrm{C}\right]$ raclopride $\mathrm{BP}_{\mathrm{ND}}$ reduction and $\mathrm{BOLD}$ activation of the right SN/VTA (data not shown).

We had further hypothesized that $\left[{ }^{11} \mathrm{C}\right]$ raclopride displacement in the ventral striatum might correlate with fMRI activation of the same region, and tested this hypothesis with a second ROI analysis. To account for the individually variable $\mathrm{T} 2{ }^{\star}$ signal loss in the ventral striatum, individual spherical ROIs (radius $=6$ $\mathrm{mm}$ ) were centered on the subjects' individual local maxima closest to $\left[\begin{array}{lll}x & y & z\end{array}\right]=\left[\begin{array}{lll}-6 & 10 & -6\end{array}\right]$, the voxel showing the maximal radioligand displacement in the PET data (Fig. 3, left; see Fig. 4, bottom left, for a representative ROI) (see Materials and Methods for details). A linear regression analysis revealed a moderate, positive relationship between the $\mathrm{BP}_{\mathrm{ND}}$ difference (neutral - rewarded) in the left NAcc and fMRI activation of the left NAcc during presentation of reward-predicting cues (Fig. 4, bottom right).

To investigate whether NAcc dopamine release might also correlate with fMRI activations beyond the midbrain and ventral striatum, an exploratory voxelwise linear regression analysis ( $p<0.005$, uncorrected, $k=15$ voxels) was performed over the entire volumes scanned. This voxelwise analysis confirmed the result of the ROI-based statistics, revealing a significant positive correlation of $\left[{ }^{11} \mathrm{C}\right]$ raclopride $\mathrm{BP}_{\mathrm{ND}}$ reduction with $\mathrm{BOLD}$ activations in the left SN/VTA and in the left NAcc [supplemental Fig. 1 (top), available at www.jneurosci.org as supplemental material]. Additionally, we observed a positive correlation between NAcc $\left[{ }^{11} \mathrm{C}\right]$ raclopride $\mathrm{BP}_{\mathrm{ND}}$ decrease and the hemodynamic reward anticipation responses in the left amygdala and in the bilateral hippocampus as well as portions of the thalamus and dorsal striatum [supplemental Fig. 1 (bottom), available at www. jneurosci.org as supplemental material]. The only brain region in which a negative correlation of the BOLD response to reward cues with [ $\left.{ }^{11} \mathrm{C}\right]$ raclopride displacement was observed at the chosen statistical threshold was the left fusiform gyrus $\left(\left[\begin{array}{lll}x & y & z\end{array}\right]=\right.$ $[-44-46-20])$.

\section{Discussion}

Our results demonstrate that, in young healthy adults, dopamine release in a rewarded task correlates with the hemodynamic reward anticipation response in the SN/VTA, where the cell bodies of the dopaminergic neurons are located, as well as the ventral striatum, a primary target region of these neurons.

\section{Dopamine release and SN/VTA activation}

Several recent fMRI studies have recorded hemodynamic responses from the SN/VTA (Elliott et al., 2003; Wittmann et al., 2005; Bunzeck and Düzel, 2006; Dreher et al., 2006; O'Doherty et al., 2006; Schott et al., 2006) and discussed whether such responses could be a signature of dopaminergic neurotransmission. Our findings now provide evidence for a quantitative coupling between the individual BOLD response in the SN/VTA and individual variability in activity-dependent dopamine release.

The SN/VTA response to reward-predicting cues is a fast, transient burst firing process superimposed on the considerably lower baseline firing of SN/VTA neurons (Schultz, 1998). Eventrelated fMRI measures of SN/VTA activity elicited by rewardpredicting cues might reflect this burst firing, as might the observed reward-related tracer displacement. However, because $\left[{ }^{11} \mathrm{C}\right]$ raclopride PET cumulates data across a large number of events, the possibility remains that tonic background firing of SN/VTA neurons may also have contributed to reward-related $\mathrm{BP}_{\mathrm{ND}}$ changes. The observed correlation between $\mathrm{BP}_{\mathrm{ND}}$ reduction and SN/VTA fMRI signal might then be explained indirectly by the SN/VTA characteristic that only tonically active SN/VTA neurons can burst-fire in response to transient events (O’Doherty et al., 2006).

Note that positive feedback might have led to prediction error-related dopamine release, and, similarly, negative feedback might have resulted in a dip in baseline dopamine release (Schultz, 1998). Given the well learned positive expected values 


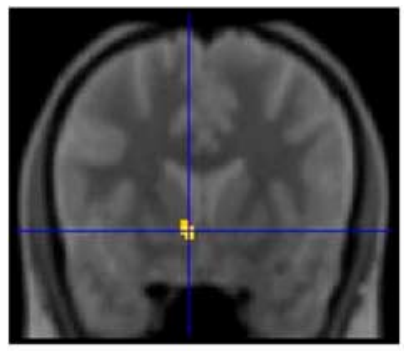

$[x$ y $z]=\left[\begin{array}{lll}-6 & 10 & -6\end{array}\right]$ $\mathrm{T}=4.96$

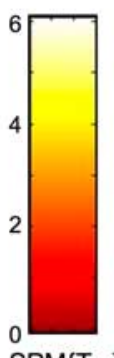

$\operatorname{SPM}\left\{\mathrm{T}_{10}\right\}$

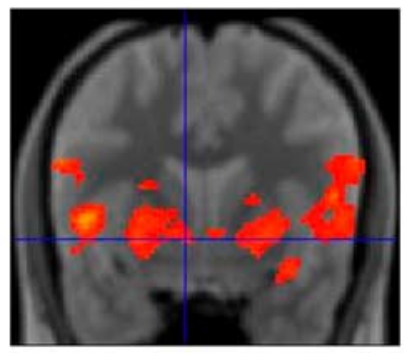

$[x$ y $z]=\left[\begin{array}{lll}-8 & 6 & -8\end{array}\right]$ $\mathrm{T}=4.33$

Figure 3. Dopamine release and fMRI activations in the ventral striatum (results from the voxel-based analysis). Left, [ $\left.{ }^{11} \mathrm{C}\right] \mathrm{ra}-$ clopride displacement in the left nucleus accumbens. Right, Activation of the ventral striatum during reward anticipation in the fMRI study. Coordinates are given in MNI space; $p<0.005$, uncorrected; extent threshold $k=15$ voxels.

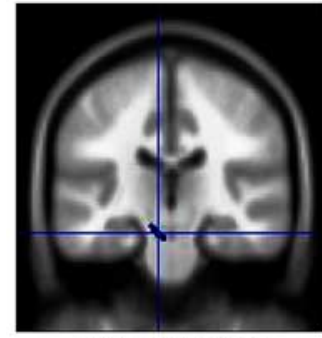

$[x y z]=[-7-23-18]$

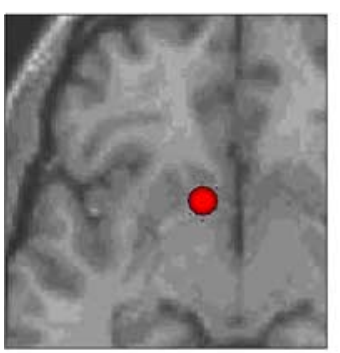

$[x y z]=\left[\begin{array}{ccc}-8 & 8 & -8\end{array}\right]$
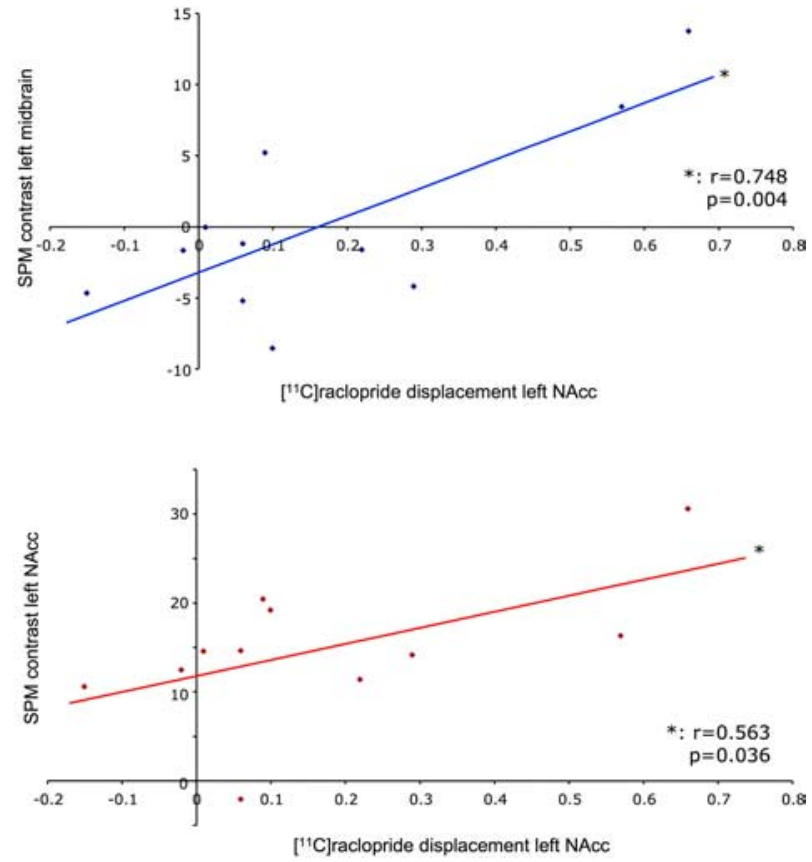

Figure 4. Correlation of dopamine release and fMRI activations. Top, Left, Location of the ROI for the left midbrain. Right, Across the study cohort, fMRI response in the left midbrain (segmented area) during reward anticipation was significantly correlated with $\left[{ }^{11} \mathrm{C}\right]$ raclopride displacement in the rewarded relative to the neutral condition. Bottom, Left, Representative ROI from a single subject. Six-millimeter spheres were centered at the local maxima of the reward anticipation response closest to $[X$ $y z]=\left[\begin{array}{ll}-6 & 10-6\end{array}\right]$ (the coordinate of maximal reward-related $\mathrm{BP}_{\mathrm{ND}}$ decrease in PET), individually for each subject. Right, $A$ significant correlation was observed between $\left[{ }^{11} \mathrm{C}\right]$ raclopride displacement and the fMRI response in the left nucleus accumbens.

associated with reward cues, it seems, however, plausible that most of the phasic dopamine release occurred during cue presentation. Any dopamine release during feedback that occurred despite the fact that we did not observe a positive fMRI prediction error during feedback would be unlikely to be substantial enough to affect our overall results.

The correlation of the SN/VTA fMRI response and dopamine release from SN/VTA neurons is noteworthy, as the BOLD signal correlates most strongly with local field potentials (LFPs), which predominantly reflect postsynaptic mechanisms (Logothetis, 2002). The hemodynamic response increase in the SN/VTA observed here-and in previous studies on human reward anticipation-might thus result to some extent from increased afferent input into the SN/VTA. Major input structures include the NAcc via the ventral pallidum (Lisman and Grace, 2005), the laterodorsal and parapontine tegmentum (Kobayashi and Okada, 2007), and the amygdala (Haber and Fudge, 1997). Given that dopa-

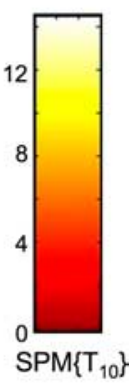

mine release also correlated with fMRI activations of the NAcc, the amygdala, and the hippocampus (Fig. 4; supplemental Fig. 1, available at www.jneurosci.org as supplemental material), we tentatively propose that the BOLD response of the SN/VTA might be a correlate of direct or indirect SN/VTA stimulation by these limbic structures. Notably, the amygdala exerts direct excitatory input to the dopaminergic midbrain (Haber and Fudge, 1997). While the amygdala has traditionally been implicated in aversive learning, animal studies have also provided evidence for a role of the amygdala in appetitive reinforcement learning (Knapska et al., 2006; Bauer et al., 2007), and human neuroimaging studies have further supported this view (Gottfried et al., 2003; Hampton et al., 2007).

\section{Regional specificity of midbrain BOLD signals}

A common neuroanatomical concept distinguishes between nigrostriatal projections from the substantia nigra, pars compacta (SNc) to the dorsal striatum (nigrostriatal system) and mesolimbic/ mesocortical pathways from the VTA to cortical and limbic structures, including the NAcc, the amygdala, and the hippocampus. Mesolimbic projections have traditionally been implicated in rewardmotivated behavior. According to this concept, midbrain activations during reward predictions should be primarily observed in the VTA, and a recent highresolution fMRI study identified a putative VTA BOLD signal related to reward prediction errors (D'Ardenne et al., 2008). Because in that study fMRI was suboptimal for the SNc, it left unclear how the SNc responded to rewards. It should be noted that the SN and VTA are highly interconnected (Ferreira et al., 2008), and that primates, particularly humans, depart from rodents by a vast expansion of the $\mathrm{SN}$ and by a distribution of mesolimbic projection neurons across the SNc (Smith and Kieval, 2000; Björklund and Dunnett, 2007; Ikemoto, 2007). These anatomical observations are mirrored by electrophysiological studies showing that dopaminergic $\mathrm{SNc}$ neurons code reward prediction errors in nonhuman primates (Ljungberg et al., 1992; Tobler et al., 2003). In the present study, we observed a lateralized correlation of striatal dopamine release with an SN/VTA BOLD signal that, as expected, extended well into the $\mathrm{SN}$. In our view, therefore, the utility of fMRI sequences optimized for specific SN/VTA compartments is limited in humans.

\section{Dopamine and the BOLD signal in the ventral striatum}

Pharmacological fMRI (Pessiglione et al., 2006) and correlations between placebo-driven dopamine release and reward sensitivity of the ventral striatum (Scott et al., 2007) have suggested a quantitative role for dopaminergic signaling in the ventral striatal re- 
ward anticipation response. Here we demonstrated that, under constant task conditions, the ventral striatal BOLD response to reward anticipation correlates positively with dopamine release in the same rewarded task. While the correlation of $\left[{ }^{11} \mathrm{C}\right]$ raclopride displacement and the SN/VTA fMRI response reflects the expected relationship between the origin and the target of dopaminergic neurotransmission, the interpretation of the correlation between radioligand displacement and fMRI activation within the NAcc is less conventional. One possible explanation would be that dopamine release might trigger a local BOLD response. Based on pharmacological MRI data, Knutson and Gibbs (2007) suggested that the BOLD signal might indeed reflect the postsynaptic effects of dopamine on striatal $\mathrm{D}_{1}$ receptors. $\mathrm{D}_{1}$ receptors are highly expressed in the NAcc and exert indirect excitatory effects via G-protein-mediated stimulation of adenylate cyclase (Hurley et al., 2001). Striatal $D_{1}$ receptors play an important role in reward-related learning processes (Tran et al., 2005; Pezze et al., 2007). The effects of synaptic dopamine on neurovascular coupling are, however, not completely understood. Choi et al. (2006) suggested that dopamine-mediated increases of regional cerebral blood volume ( $\mathrm{rCBV}$ ) in rodents might result from dopaminergic action at postsynaptic or vascular $D_{1}$ type dopamine receptors. These pharmacologically induced rCBV changes occurred over long time windows (20-60 $\mathrm{min}$ ), while the BOLD response typically lasts $\sim 15-20 \mathrm{~s}$. Because the BOLD response is closely linked to LFPs, which largely reflect postsynaptic excitatory activity (Logothetis et al., 2002), glutamatergic costimulation might also explain the correlation between striatal dopamine release and the reward-related hemodynamic response. Indeed, dopaminergic neurons form glutamatergic postsynapses (Sulzer et al., 1998) that can generate fast, excitatory signals (Chuhma et al., 2004).

An alternative explanation might be an indirect relationship between dopamine release and the NAcc BOLD response, resulting from NAcc-mediated stimulation of the SN/VTA. The NAcc exerts indirect excitatory influence on the midbrain by phasically inhibiting GABAergic ventral pallidum neurons that tonically inhibit the SN/VTA (Grace et al., 2007). The amygdala and hippocampus, reward-related activation of which also correlated with dopamine release (supplemental Fig. 1, available at www. jneurosci.org as supplemental material), might be candidate structures that stimulate the NAcc (Lisman and Grace, 2005). It can thus not be excluded that increased dopamine release might be the consequence rather than the cause of increased neural activity of the ventral striatum.

A limitation of our study is that all correlations between dopamine release and BOLD signal changes were observed at group level, as radiation safety did not allow for within-subject parametric modulations of reward probability or magnitude across a larger number of PET sessions. In our view, however, the observed parametric correlations between dopamine release and fMRI responses across subjects still speak for a quantitative relationship between released dopamine and the neural responses of the midbrain and NAcc.

\section{Clinical implications}

The positive relationship between NAcc dopamine release and reward-related mesolimbic fMRI activations was found in young healthy individuals. The situation in patients with neurological or psychiatric disorders might, however, differ in a disease-specific manner. Reward-based learning in Parkinson's patients is enhanced by the dopamine precursor L-dopa (Frank et al., 2004), suggesting a similar relationship between striatal dopamine and reward responsiveness. Similarly, attention deficit/hyperactivity disorder (ADHD) patients show reduced methylphenidateinduced dopamine release (Volkow et al., 2007) and a blunted mesolimbic reward anticipation response (Ströhle et al., 2008). Patients with schizophrenia, however, exhibit reduced mesolimbic reward anticipation responses (Juckel et al., 2006), but increased pharmacologically induced dopamine release relative to healthy controls (Breier et al., 1997), possibly due to an imbalance between phasic and tonic dopaminergic activity (Goto et al., 2007).

\section{Conclusions}

Our results provide evidence for a quantitative relationship between reward-related ventral striatal dopamine release and the mesolimbic neural response to reward cues in humans, thereby extending previous observations in animals (Schultz, 1998).

Our study extends the use of multimodal PET/fMRI imaging from the correlation of ligand binding and BOLD responses in cognitive tasks (Heinz et al., 2004) to the study of the relationship between event-related neural signatures and activity-dependent changes in molecular markers (Koepp et al., 1998; Elmenhorst et al., 2007). In this approach, event-related fMRI might provide a way to overcome the temporal limitations of PET.

\section{References}

Adcock RA, Thangavel A, Whitfield-Gabrieli S, Knutson B, Gabrieli JD (2006) Reward-motivated learning: mesolimbic activation precedes memory formation. Neuron 50:507-517.

Ahsan RL, Allom R, Gousias IS, Habib H, Turkheimer FE, Free S, Lemieux L, Myers R, Duncan JS, Brooks DJ, Koepp MJ, Hammers A (2007) Volumes, spatial extents and a probabilistic atlas of the human basal ganglia and thalamus. Neuroimage 38:261-270.

Bauer EP, Paz R, Paré D (2007) Gamma oscillations coordinate amygdalorhinal interactions during learning. J Neurosci 27:9369-9379.

Björklund A, Dunnett SB (2007) Dopamine neuron systems in the brain: an update. Trends Neurosci 30:194-202.

Breier A, Su TP, Saunders R, Carson RE, Kolachana BS, de Bartolomeis A, Weinberger DR, Weisenfeld N, Malhotra AK, Eckelman WC, Pickar D (1997) Schizophrenia is associated with elevated amphetamine-induced synaptic dopamine concentrations: evidence from a novel positron emission tomography method. Proc Natl Acad Sci U S A 94:2569-2574.

Brett M, Anton JL, Valabregue R, Poline JB (2002) Region of interest analysis using an SPM toolbox (CD-ROM). Neuroimage 16.

Bunzeck N, Düzel E (2006) Absolute coding of stimulus novelty in the human substantia nigra/VTA. Neuron 51:369-379.

Cervenka S, Bäckman L, Cselényi Z, Halldin C, Farde L (2008) Associations between dopamine D2-receptor binding and cognitive performance indicate functional compartmentalization of the human striatum. Neuroimage 40:1287-1295.

Choi JK, Chen YI, Hamel E, Jenkins BG (2006) Brain hemodynamic changes mediated by dopamine receptors: role of the cerebral microvasculature in dopamine-mediated neurovascular coupling. Neuroimage 30:700-712.

Chuhma N, Zhang H, Masson J, Zhuang X, Sulzer D, Hen R, Rayport S (2004) Dopamine neurons mediate a fast excitatory signal via their glutamatergic synapses. J Neurosci 24:972-981.

D'Ardenne K, McClure SM, Nystrom LE, Cohen JD (2008) BOLD responses reflecting dopaminergic signals in the human ventral tegmental area. Science 319:1264-1267.

Dreher JC, Kohn P, Berman KF (2006) Neural coding of distinct statistical properties of reward information in humans. Cereb Cortex 16:561-573.

Elliott R, Newman JL, Longe OA, Deakin JF (2003) Differential response patterns in the striatum and orbitofrontal cortex to financial reward in humans: a parametric functional magnetic resonance imaging study. J Neurosci 23:303-307.

Elmenhorst D, Meyer PT, Winz OH, Matusch A, Ermert J, Coenen HH, Basheer R, Haas HL, Zilles K, Bauer A (2007) Sleep deprivation increases $A_{1}$ adenosine receptor binding in the human brain: a positron emission tomography study. J Neurosci 27:2410-2415.

Ferreira JG, Del-Fava F, Hasue RH, Shammah-Lagnado SJ (2008) Organi- 
zation of ventral tegmental area projections to the ventral tegmental areanigral complex in the rat. Neuroscience 153:196-213.

Frank MJ, Seeberger LC, O’Reilly RC (2004) By carrot or by stick: cognitive reinforcement learning in parkinsonism. Science 306:1940-1943.

Friston KJ, Fletcher P, Josephs O, Holmes A, Rugg MD, Turner R (1998) Event-related fMRI: characterizing differential responses. Neuroimage $7: 30-40$.

Goto Y, Otani S, Grace AA (2007) The Yin and Yang of dopamine release: a new perspective. Neuropharmacology 53:583-587.

Gottfried JA, O'DohertyJ, Dolan RJ (2003) Encoding predictive reward value in human amygdala and orbitofrontal cortex. Science 301:1104-1107.

Grace AA, Floresco SB, Goto Y, Lodge DJ (2007) Regulation of firing of dopaminergic neurons and control of goal-directed behaviors. Trends Neurosci 30:220-227.

Haber SN, Fudge JL (1997) The primate substantia nigra and VTA: integrative circuitry and function. Crit Rev Neurobiol 11:323-342.

Hampton AN, Adolphs R, Tyszka MJ, O’Doherty JP (2007) Contributions of the amygdala to reward expectancy and choice signals in human prefrontal cortex. Neuron 55:545-555.

Heinz A, Siessmeier T, Wrase J, Hermann D, Klein S, Grüsser SM, Flor H, Braus DF, Buchholz HG, Gründer G, Schreckenberger M, Smolka MN, Rösch F, Mann K, Bartenstein P (2004) Correlation between dopamine $\mathrm{D}(2)$ receptors in the ventral striatum and central processing of alcohol cues and craving. Am J Psychiatry 161:1783-1789.

Holschbach M, Schüller M (1993) A new and simple on-line method for the preparation of n.c.a. [11C]methyl iodide. Appl Radiat Isot 44:779-780.

Hurley MJ, Mash DC, Jenner P (2001) Dopamine D(1) receptor expression in human basal ganglia and changes in Parkinson's disease. Brain Res Mol Brain Res 87:271-279.

Ikemoto S (2007) Dopamine reward circuitry: two projection systems from the ventral midbrain to the nucleus accumbens-olfactory tubercle complex. Brain Res Rev 56:27-78.

Innis RB, Cunningham VJ, Delforge J, Fujita M, Gjedde A, Gunn RN, Holden J, Houle S, Huang SC, Ichise M, Iida H, Ito H, Kimura Y, Koeppe RA, Knudsen GM, Knuuti J, Lammertsma AA, Laruelle M, Logan J, Maguire RP, et al. (2007) Consensus nomenclature for in vivo imaging of reversibly binding radioligands. J Cereb Blood Flow Metab 27:1533-1539.

Josephs O, Henson RN (1999) Event-related functional magnetic resonance imaging: modelling, inference and optimization. Philos Trans R Soc Lond B Biol Sci 354:1215-1228.

Juckel G, Schlagenhauf F, Koslowski M, Wüstenberg T, Villringer A, Knutson B, Wrase J, Heinz A (2006) Dysfunction of ventral striatal reward prediction in schizophrenia. Neuroimage 29:409-416.

Knapska E, Walasek G, Nikolaev E, Neuhäusser-Wespy F, Lipp HP, Kaczmarek L, Werka T (2006) Differential involvement of the central amygdala in appetitive versus aversive learning. Learn Mem 13:192-200.

Knutson B, Cooper JC (2005) Functional magnetic resonance imaging of reward prediction. Curr Opin Neurol 18:411-417.

Knutson B, Gibbs SE (2007) Linking nucleus accumbens dopamine and blood oxygenation. Psychopharmacology 191:813-822.

Knutson B, Fong GW, Adams CM, Varner JL, Hommer D (2001) Dissociation of reward anticipation and outcome with event-related fMRI. Neuroreport 12:3683-3687.

Kobayashi Y, Okada K (2007) Reward prediction error computation in the pedunculopontine tegmental nucleus neurons. Ann NY Acad Sci 1104:310-323.

Koepp MJ, Gunn RN, Lawrence AD, Cunningham VJ, Dagher A, Jones T, Brooks DJ, Bench CJ, Grasby PM (1998) Evidence for striatal dopamine release during a video game. Nature 393:266-268.

Lammertsma AA, Hume SP (1996) Simplified reference tissue model for PET receptor studies. Neuroimage 4:153-158.

Lisman JE, Grace AA (2005) The hippocampal-VTA loop: controlling the entry of information into long-term memory. Neuron 46:703-713.

Ljungberg T, Apicella P, Schultz W (1992) Responses of monkey dopamine neurons during learning of behavioral reactions. J Neurophysiol 67:145-163.

Logan J, Fowler JS, Volkow ND, Wang GJ, Ding YS, Alexoff DL (1996) Distribution volume ratios without blood sampling from graphical analysis of PET data. J Cereb Blood Flow Metab 16:834-840.

Logothetis NK (2002) The neural basis of the blood-oxygen-level- dependent functional magnetic resonance imaging signal. Philos Trans R Soc Lond B Biol Sci 357:1003-1037.

O'Doherty JP, Buchanan TW, Seymour B, Dolan RJ (2006) Predictive neural coding of reward preference involves dissociable responses in human ventral midbrain and ventral striatum. Neuron 49:157-166.

Pappata S, Dehaene S, Poline JB, Gregoire MC, Jobert A, Delforge J, Frouin V, Bottlaender M, Dolle F, Di Giamberardino L, Syrota A (2002) In vivo detection of striatal dopamine release during reward: a PET study with $[(11) \mathrm{C}]$ raclopride and a single dynamic scan approach. Neuroimage 16:1015-1027.

Pessiglione M, Seymour B, Flandin G, Dolan RJ, Frith CD (2006) Dopamine-dependent prediction errors underpin reward-seeking behaviour in humans. Nature 442:1042-1045.

Pezze MA, Dalley JW, Robbins TW (2007) Differential roles of dopamine D1 and D2 receptors in the nucleus accumbens in attentional performance on the five-choice serial reaction time task. Neuropsychopharmacology 32:273-283.

Pietrzyk U, Herholz K, Fink G, Jacobs A, Mielke R, Slansky I, Würker M, Heiss WD (1994) An interactive technique for three-dimensional image registration: validation for PET, SPECT, MRI and CT brain studies. J Nucl Med 35:2011-2018.

Schott BH, Henson RN, Richardson-Klavehn A, Becker C, Thoma V, Heinze HJ, Düzel E (2005) Redefining implicit and explicit memory: the functional neuroanatomy of priming, remembering, and control of retrieval. Proc Natl Acad Sci U S A 102:1257-1262.

Schott BH, Seidenbecher CI, Fenker DB, Lauer CJ, Bunzeck N, Bernstein HG, Tischmeyer W, Gundelfinger ED, Heinze HJ, Düzel E (2006) The dopaminergic midbrain participates in human episodic memory formation: evidence from genetic imaging. J Neurosci 26:1407-1417.

Schott BH, Niehaus L, Wittmann BC, Schütze H, Seidenbecher CI, Heinze HJ, Düzel E (2007) Ageing and early-stage Parkinson's disease affect separable neural mechanisms of mesolimbic reward processing. Brain 130:2412-2424.

Schultz W (1998) Predictive reward signal of dopamine neurons. J Neurophysiol 80:1-27.

Scott DJ, Stohler CS, Egnatuk CM, Wang H, Koeppe RA, Zubieta JK (2007) Individual differences in reward responding explain placebo-induced expectations and effects. Neuron 55:325-336.

Smith Y, Kieval JZ (2000) Anatomy of the dopamine system in the basal ganglia. Trends Neurosci 23:S28-S33.

Ströhle A, Stoy M, Wrase J, Schwarzer S, Schlagenhauf F, Huss M, Hein J, Nedderhut A, Neumann B, Gregor A, Juckel G, Knutson B, Lehmkuhl U, Bauer M, Heinz A (2008) Reward anticipation and outcomes in adult males with attention-deficit/hyperactivity disorder. Neuroimage 39:966-972.

Stüsgen S, Lang M, Bier D (2007) A new and reliable radiosynthesis of n.c.a. [11C]raclopride. Labelled Cpd Radiopharm 50:173.

Sulzer D, Joyce MP, Lin L, Geldwert D, Haber SN, Hattori T, Rayport S (1998) Dopamine neurons make glutamatergic synapses in vitro. J Neurosci 18:4588-4602.

Tobler PN, Dickinson A, Schultz W (2003) Coding of predicted reward omission by dopamine neurons in a conditioned inhibition paradigm. J Neurosci 23:10402-10410.

Tran AH, Tamura R, Uwano T, Kobayashi T, Katsuki M, Ono T (2005) Dopamine D1 receptors involved in locomotor activity and accumbens neural responses to prediction of reward associated with place. Proc Natl Acad Sci U S A 102:2117-2122.

Volkow ND, Wang GJ, Newcorn J, Telang F, Solanto MV, Fowler JS, Logan J, Ma Y, Schulz K, Pradhan K, Wong C, Swanson JM (2007) Depressed dopamine activity in caudate and preliminary evidence of limbic involvement in adults with attention-deficit/hyperactivity disorder. Arch Gen Psychiatry 64:932-940.

Wittmann BC, Schott BH, Guderian S, Frey JU, Heinze HJ, Düzel E (2005) Reward-related FMRI activation of dopaminergic midbrain is associated with enhanced hippocampus-dependent long-term memory formation. Neuron 45:459-467.

Wittmann BC, Bunzeck N, Dolan RJ, Düzel E (2007) Anticipation of novelty recruits reward system and hippocampus while promoting recollection. Neuroimage 38:194-202.

Zald DH, Boileau I, El-Dearedy W, Gunn R, McGlone F, Dichter GS, Dagher A (2004) Dopamine transmission in the human striatum during monetary reward tasks. J Neurosci 24:4105-4112. 